\title{
Smart Home/Building Energy Management System for Future Smart Grid Architecture with Automated Demand Response Applications
}

\author{
Z. W. Cheo*, H. T. Yang*, K. Kansala\#, K. Maatta", J. Rehu\#, and W. L. Chen^ \\ *Department of Electrical Engineering, National Cheng Kung University (NCKU), \\ No. 1, University Rd, East District, Tainan City ,70101, Taiwan. \\ \#VTT Technical Research Centre of Finland Ltd, \\ P.O. Box 1100, FI-90571 Oulu, Finland. \\ $\wedge$ Smart Solution BU, Tatung Company, \\ No. 22, Section 2, Zhongshan North Rd, Zhongshan District, Taipei City, 10435, Taiwan.
}

htyang@mail.ncku.edu.tw, klaus.kansala@vtt.fi

\begin{abstract}
A home/building energy management system (H/BEMS) is the fundamental unit for smart-grid development. This paper proposes a pilot project developed for smart H/BEMS, where the load (power consumption) can be scheduled, controlled, or optimized. The proposed energy management system (EMS) integrates the Virtual End Node (VEN) for OpenADR 2.0b Alliance platform such that demand-side management can be performed automatically according to the event issued from the Virtual Top Node (VTN). Wireless sensors using Bluetooth 4.0 (Bluetooth Low Energy, BLE) as the communication protocol are also developed and integrated in the EMS architecture. Finally, the developed EMS architecture has been implemented into 24 households with smart meter each installed in public housing in Taipei, Taiwan for testing and demonstration. Results show that the proposed EMS has successfully reduced the power consumption of a household averaged about $12.62 \%$ for the past 12 months.
\end{abstract}

Keywords: Automatic demand response, demand side management, scheduling, web-based user interface, wireless sensors

\section{Introduction}

The energy consumption in home or building contributes a large portion of energy demand. According to Taiwan Bureau of Energy, $19.45 \%$ of the electricity users are from residential area [1], which consumes up to 44.9 TWh of electricity per year. At the same time, the energy consumption of residential area keeps increasing. Therefore, a home energy management system (HEMS) is required to be integrated with advanced metering infrastructure (AMI) such that large portion of energy consumption can potentially be reduced. 
On the other hand, distributed energy resources (e.g. solar photovoltaic) have been installed at an increasing rate. Excessive installation of distributed resources may have some negative impacts on the power grid, including over-voltage issue or reverse power flow. Clearly, there is a limit on the penetration of the distributed resources [2], [3]. To better manage the high penetration of distributed resources, several smart grid techniques have been proposed, like optimal management of active and reactive power control using smart inverters and load management [4]. To have a building energy management system (BEMS) is thus the fundamental technique for the power consumption management.

Several papers have been proposed on different types of energy management system. In [5], an HEMS has been proposed based on power-line communication (PLC) to handle or to control networked devices. However, optimization of the energy consumption is not considered in this work. References [6] and [7] further consider the consumption analysis for the home electrical devices, but without considering the impact of distributed renewable resources. More recent work in HEMS proposed considers both energy consumption and generation, simultaneously, to minimize the energy cost. In [8], a smart HEMS including renewable energy generation management has been proposed. The proposed system is expected to have energy-saving effects.

Some studies have been done on HEMS combined with automated demand response (ADR). In [9], the demand response (DR) programs have been shown to be able to provide regulation of demand side at critical peak hours by shifting or shedding loads. In [10], the author proposes an algorithm for HEMS combining with DR applications and studies the impact of HEMS operation on customer comfort. Currently, most of the DR studies in the literature generally consist of human interventions [10]. In [11], a dynamic control space matrix has been proposed and simulated at VTT Smart Grid Research laboratory. The proposed matrix helps to balance energy production and consumption within a building. Consider that a fully automated DR hasn't been presented in practical applications, this paper proposes a smart home and building energy management system with fully automated DR application algorithm, and discusses on the practical issues on DR event when dealing with human comfort and human decision.

The rest of the paper is organized as follows. Section 2 describes the overall system structure and its corresponding communication protocol. Section 3 describes the main function of the developed EMS. Section 4 discusses about contribution of the developed EMS and finally Section 5 concludes the paper.

\section{System Structure Corresponding Communication Protocol}

Fig. 1 shows the architecture of the system, with the corresponding communication protocols employed. The proposed H/BEMS was implemented at VTT's research apartment with the electrical appliances and devices, including dish washer, washing machine, sauna, cooking stove, floor heating and room ventilation system. The appliances and devices are all wirelessly connected to the main gateway. The smart devices are added to the apartment for intelligent controlling, including the consumption management during DR event. 
In addition, the Wireless Sensor Network (WSN) installed in the apartment consists of thirteen $2.4 \mathrm{GHz}$ Bluetooth Low Energy sensor nodes (BTLE-node) including temperature, humidity and air pressure sensor (THP-node). Further, wireless carbon dioxide $\left(\mathrm{CO}_{2}\right)$ and air pressure difference (PD) sensor are also equipped in the WSN.

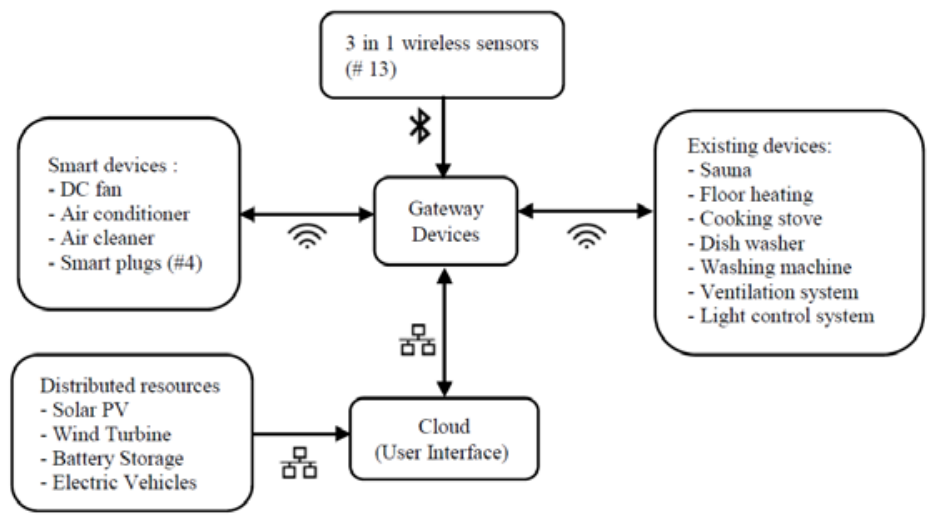

Fig. 1. System structure and corresponding communication protocol

Fig. 2 (right) shows the installation of the WSN in the apartment. The rooms, balcony and ventilation machines intake, exhaust, supply and return channels are equipped with the battery operated THP-node. $\mathrm{CO}_{2}$ and PD-nodes are installed in the living room. The BTLE-sensors utilizes the property of Bluetooth message advertising where BTLE-peripheral device broadcasts the sensor data in certain constructed packet so that the devices around can listen and receive the broadcasted messages. By advertising the sensor data, there is no need for establishing connection between the peripheral device and the receiver unit. This improves reactiveness as making a connection is much slower than advertising the message. It is also more energy efficient since the radio is on only for a short period of time during broadcasting and wide spacing of the three advertisement channels prevents interference from other $2.4 \mathrm{GHz}$ devices. The receiver gateway is a credit card size CPU with BTLE interface which then further sends the data through Wide Area Network (WAN) interface into the cloud servers.
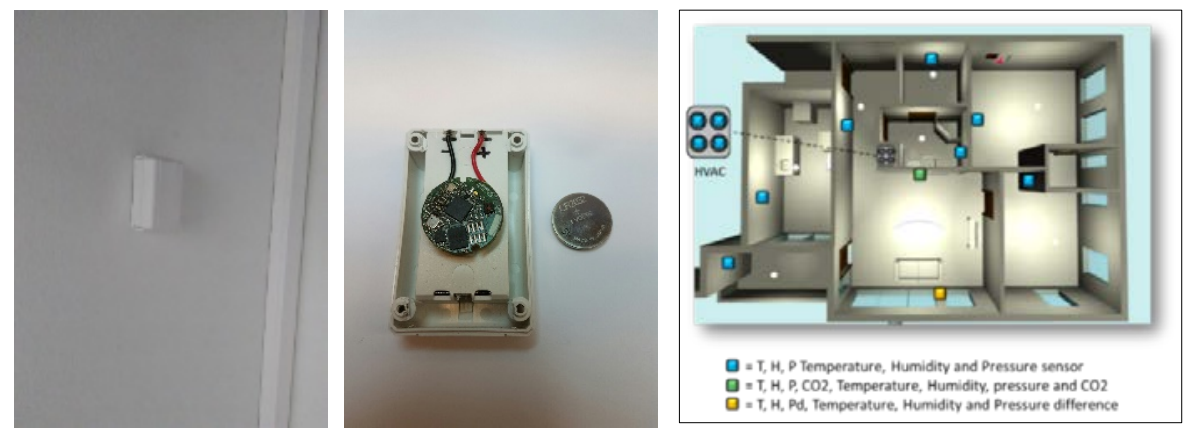

Fig. 2. VTT's Bluetooth wireless sensor and the installation of WSN in the apartment 
Finally, at the main office of VTT Oulu (right beside the apartment), distributed resources including $7.2 \mathrm{~kW}_{\mathrm{p}}$ of solar PV, $5.5 \mathrm{~kW}$ of small scale wind turbine, $58 \mathrm{kWh}$ of energy storage system (ESS) with $400 \mathrm{~V} 3$-phase $15 \mathrm{~kW}$ inverter and two electric vehicles charging posts are installed. These devices are connected to the cloud using Ethernet cable. The power generation or consumption data are stored in the cloud for further data analysis. Fig. 3 (left) shows the VTT’s local energy production test site.

\section{Key Functions Developed for Energy Management}

The proposed EMS contains four main energy management functions, as will be discussed in the following sections.

\subsection{Remote Monitoring and Control}

The smart devices can be controlled remotely using the user interface (UI) developed (Fig. 3 right). The proposed system provides automation of smart devices according to user-defined input. If a device is set to function automatically, it will only operate when the corresponding user-defined parameters have been triggered. For example, the existing ventilation system will function according to (1)

$$
\text { Mode }= \begin{cases}\text { On } & \text { if } \mathrm{C}_{\mathrm{CO}_{2}}>\text { upper limit } \\ \text { off } & \text { if } \mathrm{C}_{\mathrm{CO}_{2}}<\text { lower limit }\end{cases}
$$

where $\mathrm{C}_{\mathrm{CO}_{2}}$ is the concentration of carbon dioxide $\left(\mathrm{CO}_{2}\right)$ (measured by the WSN). The upper and lower limit are user-defined parameters. The same principle is applied to the other smart devices in the apartment.

The intelligent Light Control System (LCS) installed in the apartment is distributed using CAN-bus based system, which consists of outputs for all lamps and inputs for every switch and sensors. LCS consists of motion and light sensors for every room. VTT's gateway allows control interfaces to the LCS and produces current measurements for every lamp.
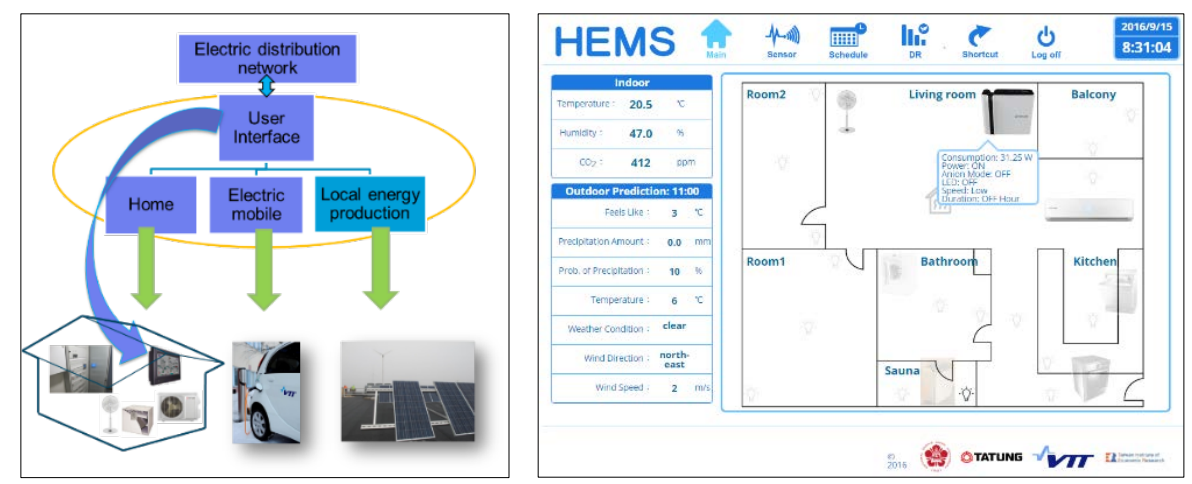

Fig. 3. VTT's local energy production test site and HEMS user interface 
Monitoring of the data from the distributed resources (solar PV, wind mill, ESS, and electric vehicle charging posts) is another important function. These information is required since schedulable load like electric vehicles or ESS can be scheduled to connect to the grid for fast charging or discharging. The Ethernet cable communication is now set to exchange data every minute (Fig. 4), with the data recorded to the database.
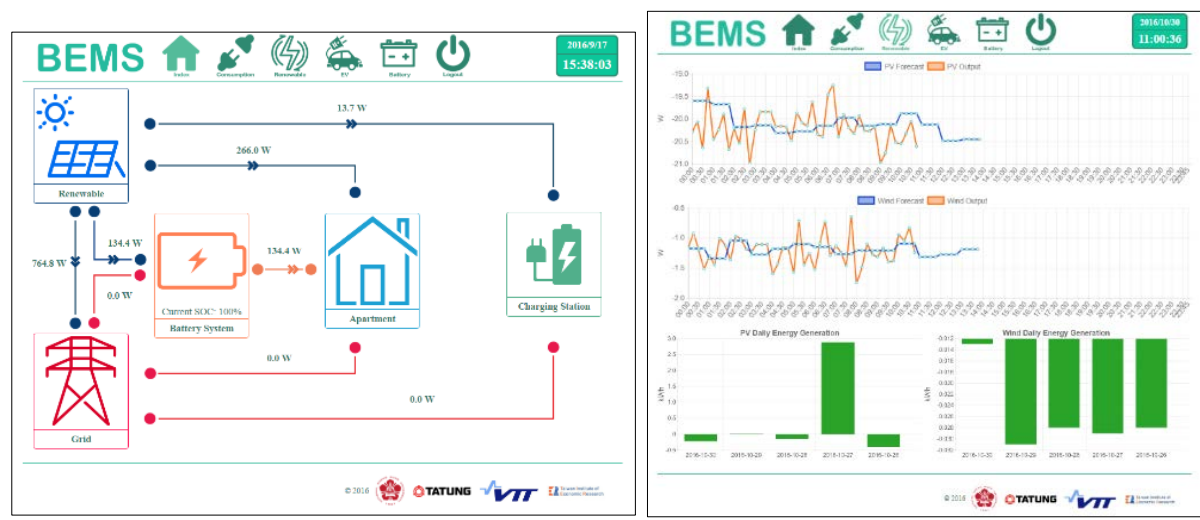

Fig. 4. BEMS real-time monitoring and renewable (solar and wind) generation record

\subsection{Smart Scheduling of Appliances}

The proposed HEMS in the literatures mainly simulated smart scheduling by using a few assumptions including users' comfort or users' requirement; however, in the proposed EMS, these kind of user parameters are given to the users to define. The proposed algorithm will start to give scheduling when the parameters on the user interface has been changed. In Fig. 5 (left), the proposed EMS algorithm schedules the devices such that the electricity cost will be minimized. The objective function and the required limiting functions are shown in (2).

Objective function: $\min \left(B_{24}\right)$

subject to

$$
B_{24}=\sum_{1}^{a} \sum_{t=t_{s}}^{t_{e}} p_{t} \times C_{\text {avg }}
$$

$$
\begin{aligned}
& t_{e} \leq \text { end time } \\
& t_{s} \geq \text { start time }
\end{aligned}
$$

where $B_{24}$ is the total amount of electricity cost for the next 24 hours. $p_{t}$ is the electricity price at time $t . C_{a v g}$ is the average power consumption of appliance $a$. In this paper, the value of $C_{a v g}$ for each appliances was obtained by averaging the power consumption for one normal operation period. $a$ is the number of appliance while $t_{e}$ and $t_{s}$ are the end time and start time of each appliance (defined by the user).

Different power contracts can also be compared (Fig. 5 lower left). Users can select the appropriate power contract that best suits the users' power usage pattern. In the proposed EMS project, the tariff information was obtained from Helen Ltd's official website. In the next phase of the project, the electricity tariff is going to be updated 
dynamically using Nord Pool electricity market. The results shown in the user interface can therefore be updated every time when the price information is updated.

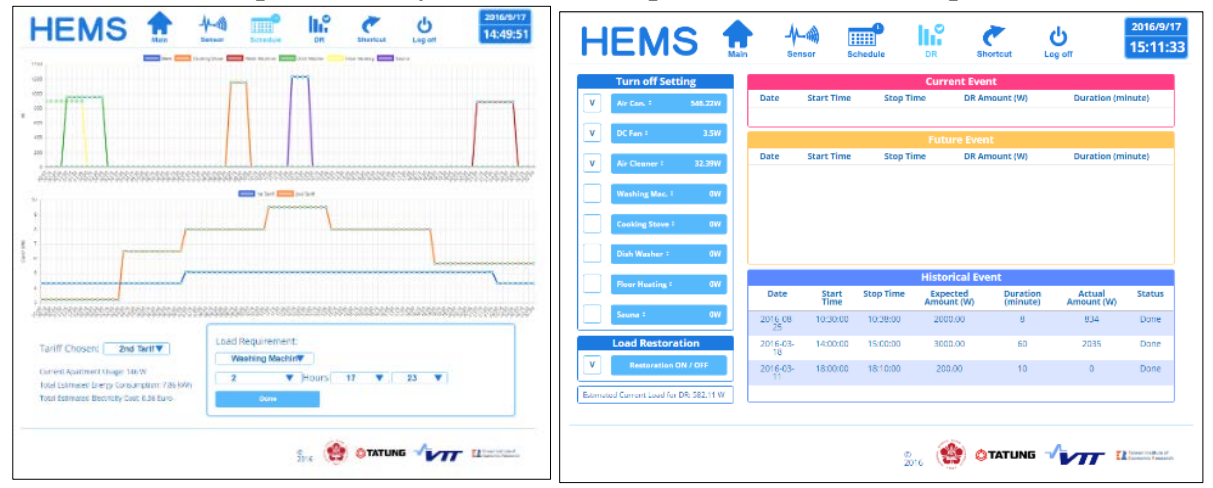

Fig. 5. Devices scheduling and demand response

\subsection{Automatic Demand Response}

The proposed EMS integrates the VEN (Virtual End Node) server in the International OpenADR 2.0b Alliance. The VEN server automatically extracts the DR event generated by the power company or center aggregators (VTN, Virtual Top Node) where the detailed information will be passed to the HEMS user interface for the user. Normally, the current VEN server algorithm checks for DR event every one hour. It is possible to check for DR event every 15-minutes during peak period. In the proposed algorithm, 15-minutes is set as the minimum checking frequency. During DR event, the proposed EMS will cut off the available and controllable loads by trying to meet the demand required by the VTN. The block diagram of the DR event is shown in Fig. 6 .

Beside the proposed DR algorithm is fully automatically executed, it is also different from the DR algorithm proposed in the literature, as listed below:

1. Users can decide whether or not to participate in the DR event, by selecting the devices to contribute to the event.

2. The amount of load shedding is calculated according to the actual amount of power consumption of the devices; the consumption data is measured using smart plug for each device.

3. Users can decide the order of the device list during DR event; the proposed EMS cuts off power supply of the devices according to the sequence in the device list. This helps to minimize the discomfort to the users.

4. Users can select whether to abstain from the DR event. If any of the devices is removed from the device list during DR event, the EMS algorithm will automatically restore the device to its original state. Users are therefore removed from the award list for participating in DR event.

5. After the DR event, the EMS will restore the affected device to the original state before DR event. Users can also select whether to or not to restore the affected devices. For example, if the user would like to leave the home during DR event, then the user would select not to restore the affected devices after the event has finished. 


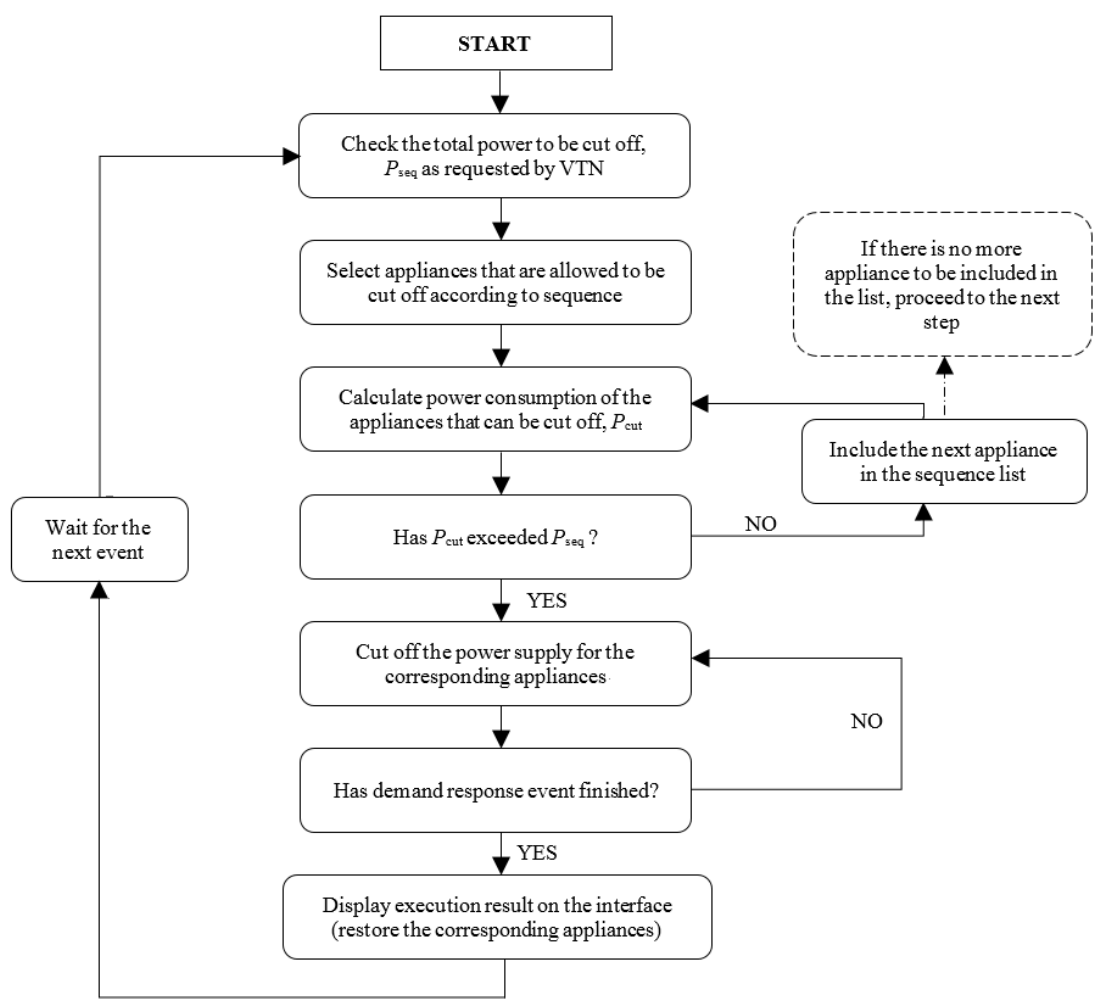

Fig. 6. The flow chart of demand response

\subsection{Power Consumption Analysis}

In Fig. 7, the developed EMS provides three important information for the users. First, "Historical Energy Usage Pattern" indicates how much electricity the user has been using each day. Second, "Current Power Usage Distribution" shows where the power is being used at the moment. It is worth to mention about the power usage amount of the item "others". The power usage of "others", $P_{\text {others }}$ is calculated using (5)

$$
P_{\text {others }}=P_{\text {total }}-\sum_{a=1}^{n} P_{\text {appliances }}
$$

where $P_{\text {total }}$ is the total power consumed by the apartment at the moment. $P_{\text {appliances }}$ is the power consumed by each of the appliances and $n$ is the number of appliances in the apartment. If $P_{\text {others }}$ is always showing a significant number, that means there must be some other unknown appliances which are consuming power. It is also possible that there can be power leakage in the apartment where inspection of the electrical system should be done. Finally, "Power Usage Today" allows the user to know the accumulated electricity that has been used for each appliances at the day. The above information allows the user to know the electricity consumption pattern and allows the users to try to minimize electricity consumption. 

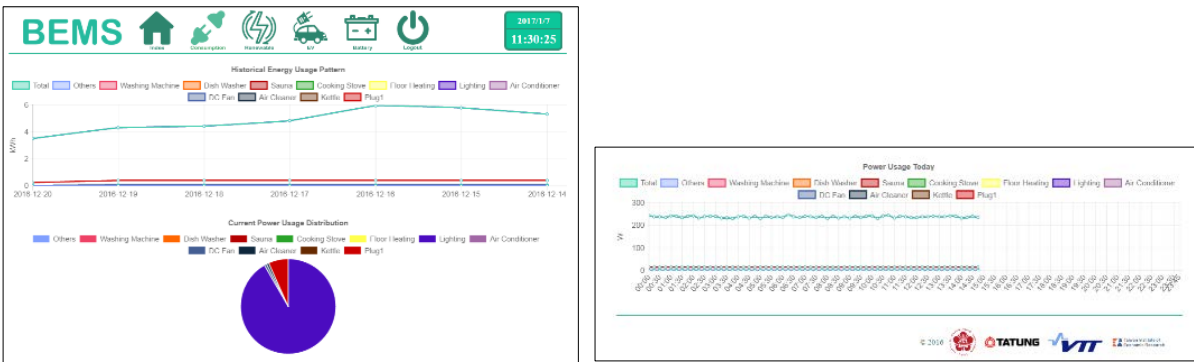

Fig. 7. The user interface of power consumption analysis

\section{Contribution of the Developed H/BEMS System}

In the National Energy Project (NEP) in Taiwan, the developed energy management system was implemented into 24 households (out of 272 households) in Xinglong public housing $1^{\text {st }}$ block (18 floors in total). The developed EMS includes visualization of energy usage (for each household) and also simple remote controlling of lightings and air-conditioners. The project was launched at March, 2016.

From the launching date until March, 2017 (one year around), the electricity usage for households with and without HEMS installed are analyzed and compared. The daily average electricity usage of the 24 households with HEMS and the next 24 households without HEMS are calculated with the following limiting functions:

$$
\begin{gathered}
E_{\text {avg }}-3 \sigma<E_{\text {daily }, n}<E_{\text {avg }}+3 \sigma \\
E_{\text {daily }, n} \geq 1
\end{gathered}
$$

Equation (6) indicates that the if the daily electricity usage for household $n, E_{\text {daily,n }}$ lies outside \pm three standard deviations, $3 \sigma$, then it is considered as an outlier which shouldn't be included in the average values. Equation (7) indicates that if the daily electricity consumption is less than $1 \mathrm{kWh}$, this implies that the householder might not be at home and hence the corresponding data is not included in the average values.

Fig. 8 shows the daily electricity usage and corresponding electricity saving households with and without HEMS installed. As can be seen, households with HEMS installed achieve positive energy saving most of the days throughout the whole period. The overall energy saving is calculated using mean absolute percentage error (MAPE) and result shows that total energy can be saved with HEMS installed is $12.62 \%$.

At the same time, the developed automated DR algorithm was also implemented at Taipower Research Institute (TPRI) Shulin Campus in Taiwan. As one of the successful seven DR events practically implemented in year 2016, shown in Fig. 9 is a realistic DR event with about 60kW of load (electrical appliances) shed throughout BEMS for two hours on $19^{\text {th }}$ July 2016 from 13:30 to 15:30. During the event, $50 \mathrm{~kW}$ of power was also discharged from the ESS controlled by the proposed EMS to achieve the targeted goal of DR with less impact on the customers. With large number of the developed EMS in households/buildings, it is expected to have more than hundreds of $\mathrm{kW}$ demand side management available in the grid of a community. The demand side management via the developed EMS may become an effective tool in maintaining power balance of the grid, especially during peak hours. 


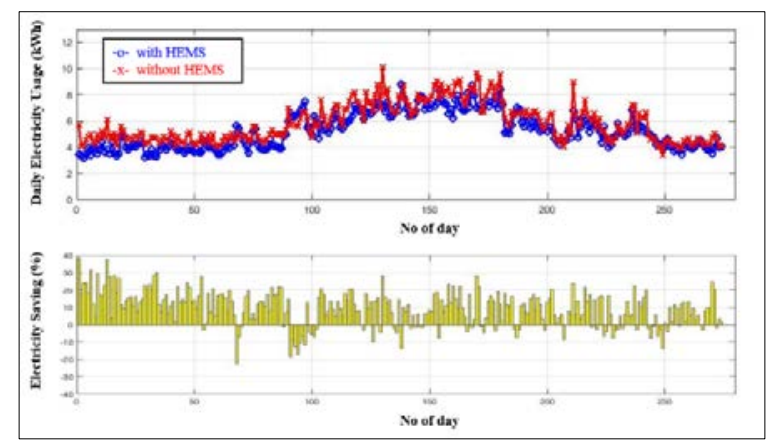

Fig. 8. Daily electricity usage and corresponding electricity saving

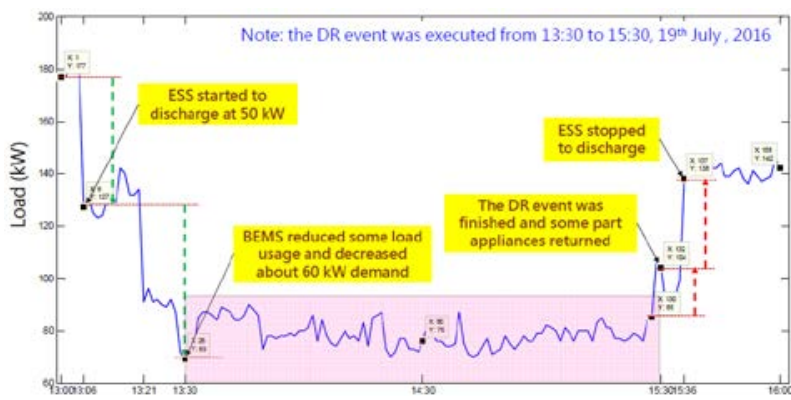

Fig. 9. Load profile during the DR event at TPRI Shulin campus on $19^{\text {th }}$ July 2016

Finally, the wireless sensor network developed by VTT under the smart home architecture has also been proved to be a successful solution. As the sensor network is critical, the $2.4 \mathrm{GHz}$ Bluetooth Low Energy sensor node help to eliminate the complex wiring of the sensors. Users can add (remove) or relocate the sensors easily, which largely promote the development of smart home to the public.

\section{Conclusion and Future Prospects}

Energy management system is the essential basic element in smart grid architecture. VTT's demonstration project has successfully shown how a basic building block of EMS can be installed, with the controlling algorithm developed in the paper. There are a few items to be improved or to be included in the next phase of the project.

1. With smarter appliance developed, it is possible to foresee the power usage pattern throughout its whole operating period. Hence, the estimated power consumption can be more accurately monitored.

2. The proposed algorithm can be applied to real-time or spot-pricing power market. In a smart grid architecture, power company or market may broadcast real-time pricing information about the real power and auxiliary service demands via the AMI system. The EMS may react and provide dynamic load control as requested based on the preference defined by the users ahead of time. 
3. Besides the load shedding during demand response event, the proposed system can provide dynamic voltage or frequency control over short term period with smart devices (e.g. smart inverter) installed.

Finally, the proposed system can be extended to the other different units, like supermarket, commercial offices, or industrial sectors, which is under planning in the next phase of the project.

\section{Acknowledgements}

This work was co-sponsored by Tatung Company and the Ministry of Science and Technology, Taiwan, under Grant MOST 105-3113-E-006-007.

\section{References}

1. Taiwan Bureau of Energy, Ministry of Economic Affairs; https://www.moeaboe.gov.tw/ECW/populace/content/ContentLink.aspx?menu_id=378 [accessed at 2017-01-07].

2. D. M. Francisco and D. M. Andrew, "Endogenous assessment of the capacity value of solar PV in generation investment planning studies," IEEE Trans. Sustainable Energy, vol. 6, no. 4, pp. 1574-1585, Oct. 2015.

3. D. W. Jeremy, R. W. Neville, S. M. David, R. W. Alan, L. Scott, and J. V. Allan, "Impact of solar photovoltaics on the low-voltage distribution network in New Zealand," IET Generation, Transmission and Distribution, vol. 10, no. 1, pp. 1-9, Aug. 2015.

4. F. Olivier, P. Aristidou, D. Ernst, and T. V. Cutsem, "Active management of low-voltage networks for mitigating overvoltages due to Photovoltaic units,” IEEE Trans. Smart Grid, vol. 7, no. 2, pp. 926-936, Mar. 2016.

5. Y. S. Son, T. Pulkkinen, K. D. Moon, and C. Kim, "Home energy management system based on power line communication,” IEEE Trans. Consumer Electronic, vol. 56, no. 3, pp. 13801386, Aug. 2010.

6. J. Han, C.S. Choi, W. K. Park, and I. Lee, "Green home energy management system through comparison of energy usage between the same kinds of home appliances,", in 2011 IEEE $15^{\text {th }}$ International Symposium on Consumer Electronics, Singapore, Jun. 2011.

7. C. H. Lien, H. C. Chen, Y. W. Bai, and M. B. Lin, "Power monitoring and control for electric home appliances based on power line communication," in IEEE International Instrumentation and Measurement Technology Conference, Vancouver, Canada, May 2008

8. J. Han, C. S. Choi, W. K. Park, I. Lee, and S. H. Kim, "Smart home energy management system including renewable energy based on ZigBee and PLC," IEEE Transactions on Consumer Electronics, vol. 60, no. 2, pp. 198-202, May 2014.

9. B. Kladnik, G. Artac, and A. Gubina, "An assessment of the effects of demand response in electricity markets,” International Trans. Electrical Energy Systems, vol. 23, no. 3, pp. 380391, Apr. 2013.

10. M. Kuzlu, "Score-based intelligent home energy management (HEM) algorithm for demand response applications and impact of HEM operation on customer comfort," IET Generation, Transmission and Distribution, vol. 9, no. 7, pp. 627-635, Nov. 2014.

11. K. Kansala, "Virtual Grid - How to Balance Energy Production and Consumption with the Help of Intelligent Buildings," International Conference on Environment \& Electrical Engineering, Florence, Italy, Jun. 2016. 